\title{
Study on the Main Factors of Frequency Variation of LC Resonant Prestress Detection Method
}

\author{
Benniu Zhang $\mathbb{C D}^{1}{ }^{1}$ Yuanyuan Zhao ${ }^{D},{ }^{1}$ Xingxing $\mathrm{Li}^{,}{ }^{1}$ Yanpeng $\mathrm{Su},{ }^{1}$ Qiang Yin, ${ }^{2}$ \\ and Jianhui Jiang ${ }^{2}$ \\ ${ }^{1}$ Chongqing Jiaotong University, Chongqing 400074, China \\ ${ }^{2}$ Xinjiang Communications Construction Group Co., Ltd., Urumchi 830000, China
}

Correspondence should be addressed to Benniu Zhang; 990020030755@cqjtu.edu.cn

Received 26 October 2019; Accepted 11 December 2019; Published 14 January 2020

Academic Editor: Luigi Di Benedetto

Copyright ( $(2020$ Benniu Zhang et al. This is an open access article distributed under the Creative Commons Attribution License, which permits unrestricted use, distribution, and reproduction in any medium, provided the original work is properly cited.

\begin{abstract}
Stress loss of prestressed steel strands of existing bridges influences their bearing capacity, so it is of great significance to realize the stress detection. A steel strand that has an inductive property is designed into the resonant circuit, which can realize the stress measurement of the steel strands by testing the resonant frequency. This method is a promising approach for the stress detection of the steel strands. Previous research found that structural stress made the permeability of steel strands change due to the influence of magnetoelastic effect. In the process, the length of steel strands is also changed. Therefore, further research needs to be done to verify the main influence parameter affecting the resonant frequency of the circuit. Furthermore, it is very important to know how the stress affects the resonant frequency to realize the detection of the prestressed force of the steel strands. Therefore, in this paper, the relationship between stress and relative permeability and length is analyzed theoretically, and the theory of stress frequency of steel strands is modified and verified by experiments. The stress-frequency experiments of steel strands and aluminum strands with great difference in relative permeability are carried out. Experiments on stress frequencies of 7- $\Phi 15.20 \mathrm{~mm}$ steel strands with different lengths are carried out. The influence of length and permeability parameters on resonance frequency is analyzed. The experimental results show that under the same conditions, the resonant frequencies of steel strands and aluminum strands are almost the same on LC electric circuits, and the resonant frequency decreases linearly with the increase of the natural length of the component and increases linearly with the increase of stress. Consequently, compared with the influence of length change on LC electromagnetic resonance frequency, the relative permeability of the stress change component can be ignored. The stress changes the resonant frequency mainly by changing the length of the strands.
\end{abstract}

\section{Introduction}

Prestressed concrete structure is to use steel strand to exert pressure on the structure before it is subjected to external load, and the resulting prestressed state is used to reduce or offset the tensile stress caused by the external load. Because the prestressing concrete bridges take advantage of the compressive performance of concrete, while perfectly avoiding the disadvantage of poor crack resistance, it has been widely used in long-span bridges all over the world. However, for the shrinkage and creep of concrete, steel relaxation and other factors will lead to loss of prestressing force of steel strands. Prestress loss threatens the durability and serviceability of structures. Therefore, it is of great significance to detect the stress of steel strands in prestressed concrete bridges. At present, many nondestructive testing methods have been tried to apply to the prestress detection of steel strands, but no one has successfully applied to the stress detection of steel strands of prestressed concrete bridges.

Fiber Bragg grating (FBG) method can monitor the stress history and spatial distribution of prestressing steel strands. Shin et al. [1] used four different sensors to carry out experimental research on stress monitoring of steel strands. Huynh et al. [2] used this sensor to study the stress loss in the prestressed concrete structure caused by temperature. 
Experiments show that the use of fiber Bragg grating embedded sensors can effectively evaluate the stress loss of steel strands. But, it needs to be embedded in the concrete structure in advance, which has limitations for the detection of the stress loss of the steel strands of the existing prestressed bridge; ultrasound-guided wave method $[3,4]$ is a common nondestructive testing method, which is widely used to detect crack lines on metal structures and corrosion defects on steel plates. Qian et al. [5] carried out experimental research on the relationship between the stress of steel strands and the guided wave and proved that there is a good correlation between the tension and the identification index. However, due to the loss of the ultrasonic guided wave when it passes through concrete, it can only be used in grouting bonded prestressing steel. Magnetoelastic method estimates stress by measuring the magnetic properties of supermagnetic materials. Duan et al. [6-11], based on magnetoelastic effect, has carried out an experimental study on stress monitoring of the high-strength steel wire, reinforced bar, and prestressed steel strands. Based on this method, Joh et al. [12] conducted a feasibility study on noncontact stress detection of steel strands. Kim et al. [13] proposed a tension monitoring system of a prestressed beam based on an embedded electromagnetic sensor. However, the magnetoelastic method needs to be demagnetized many times before stress measurement. It is difficult to ensure consistency between the state after demagnetization and the initial state. In addition, the magnetic flux leakage method [14] can be used to estimate the stress of external prestressing tendons, but it is difficult to apply to the stress detection of steel strands wrapped in concrete.

The steel strand stress detection of the LC resonant circuit is to connect both ends of the steel strands into the circuit through the conductor. The change of the stress of the steel strands leads to the change of the inductance characteristic of the steel strands, which affects the resonance frequency of the circuit.

It has been proved that the stress of strands has a good correlation with the electromagnetic resonance frequency $[15,16]$. It is feasible to detect the prestress of strands based on the LC resonant circuit. In the early stage, Zhang et al. [17] carried out experimental research on $1.2 \mathrm{~m}$ steel strands. It was proved that the stress of steel strands has a good negative linear relationship with electromagnetic resonance frequency. It is feasible to detect the prestress of steel strands based on the LC resonant circuit. It was found that the stress of $10 \mathrm{~m}$ long steel strands is positively correlated with the resonance frequency in the later study. In order to prove this conflict, experimental study was carried out on the $1.2 \mathrm{~m}$, $10 \mathrm{~m}$, and $15 \mathrm{~m}$ steel strand and established two sets of theoretical analysis to study the length effect based on the LC resonant electric circuit. As a kind of ferromagnetic material, according to the magnetoelastic properties of the material, the change of stress will lead to the change of permeability $[15,16]$. At the same time, considering the principle of material elasticity, we know that the length of steel strands will also change. However, it is still unknown which parameter of relative permeability and length is the main factor affecting the resonant frequency. In this paper, research on this problem is carried out in order to provide guidance for the later detection methods of LC resonant prestress.

Based on the research, this paper theoretically analyzes the relationship between stress and relative permeability and length, and verifies it by experiments.

In the experiment, the steel strands $\left(u_{\mathrm{r}}=1500\right)$ and aluminum strands $\left(u_{\mathrm{r}}=1\right)$ with great difference in relative permeability are selected for stress-frequency test. The resonant frequency with the same stress and length of 7 $\Phi 9 \mathrm{~mm}$ steel strands/aluminum strands are measured by experiment, and the influence of permeability on the frequency of the LC resonant circuit is analyzed. In addition, the length frequency and stress-frequency experiments are carried out on the 1,860 steel strands of 7- $\Phi 15.2 \mathrm{~mm}$, which is commonly used in the project. The influence of the length of steel strands on the frequency of the LC resonant circuit is analyzed. According to the experimental results, the stress of the strands mainly affects the resonant frequency of the circuit by changing the length of the strands. Compared with the influence of the length, the influence of the change of permeability on the resonant frequency of the circuit can be neglected.

\section{Theoretical Model}

2.1. Introduction of Resonance Circuit. Based on the LC resonant circuit, an experimental study on stress detection of steel strands is carried out in this paper. The circuit model diagram is shown in Figure 1, the initial inductance in the circuit is simplified to a fixed inductance, and steel strands are simplified as an inductance element. They are connected in series with the inherent inductance in the circuit and in parallel with the capacitor. In the resonance circuit, there is energy resonance between inductance and capacitance. That is to say, when discharging, the electric energy in the capacitor is converted into the magnetic energy in the inductor, and when charging, the magnetic energy in the inductor is converted into the electric energy in the capacitor, forming the electromagnetic resonance relationship.

2.2. Derivation of Resonant Frequency. As shown in Figure 1, the voltage $V_{\mathrm{C}}$ at both ends of the capacitor is equal to the voltage $V_{L}$ at both ends of the total inductance due to the parallel connection of inductance and capacitance. Because in

$$
V_{C}=V_{L}
$$

the resonant circuit, the principle of energy resonance exists between inductance and capacitance; electric energy in capacitance and magnetic energy in inductance form electromagnetic resonance. External power supply continuously supplements the energy consumption in the circuit. Thus, the current flowing into the inductor is equal to the current flowing into the capacitor:

$$
i_{C}=-i_{L}
$$

From the constitutive relation of circuit elements, it can be seen that 


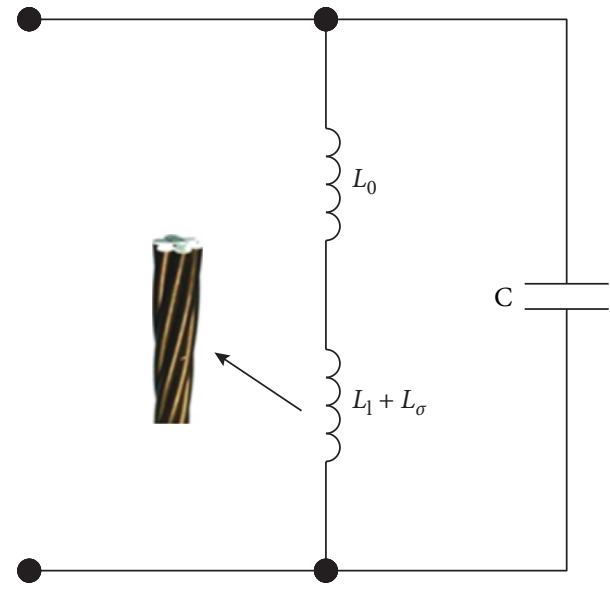

FIgURE 1: LC resonant circuit model diagram.

$$
\begin{aligned}
V_{L} t & =L \frac{\mathrm{d} i_{L}}{\mathrm{~d} t}, \\
i_{C} t & =C \frac{\mathrm{d} V_{C}}{\mathrm{~d} t}, \\
i_{C} t & =C\left(L \frac{\mathrm{d}^{2} i_{L}}{\mathrm{~d} t^{2}}\right)=C L \frac{\mathrm{d}^{2} i_{L(t)}}{\mathrm{d} t^{2}}, \\
\frac{\mathrm{d}^{2} i_{L(t)}}{\mathrm{d} t^{2}}+\frac{1}{L C} i_{L} t & =0 .
\end{aligned}
$$

The current expression is as follows:

$$
i_{L} t=C_{1} \cos \frac{1}{\sqrt{L C}} x+C_{2} \sin \frac{1}{\sqrt{L C}} x .
$$

The current angular frequency is

$$
w=w_{0}=\frac{1}{\sqrt{L C}}
$$

The resonant frequency of the circuit is

$$
f=f_{0}=\frac{1}{2 \pi \sqrt{L C}} \text {. }
$$

2.3. Inductance Deduction. As a ferromagnetic material, considering the elastic principle of the material, the length of steel strands changes under the action of stress. At the same time, the change of length will also change its magnetic properties [15]. Through the analysis and study of the steel strand structure, we can see that because the strands are made of six outer wires twisted around the central wire, it is simplified to a spiral coil model [17]. At the same time, there is a high-speed conversion between electric energy and magnetic energy between the capacitance and the test piece, simplifying the strands into a high-frequency AC circuit. According to the skin effect of the current, the current distributes on the surface of six strands around the strands, and the magnetic field inside the strands is approximately 0 . The magnetic field along the axis of a long wireless solenoid is uniform, and its values are $\mu_{0} \mathrm{NI} / l$.

$$
\begin{aligned}
B & =\frac{\mu_{0} \mathrm{NI}}{l}, \\
\Psi & =\mathrm{NBS}=\frac{\mu_{0} N^{2} \mathrm{SI}}{l}, \\
L & =\frac{\Psi}{I}=\frac{\mu_{0} N^{2} S}{l}, \\
N_{1} & =\frac{l}{a}, \\
N & =6 N_{1} .
\end{aligned}
$$

In these formulas, $\Psi$ is the magnetic flux, $S$ is the crosssection area of steel strands, $N$ is the number of coil turns of steel strands, $N_{1}$ is the number of coil turns of the single steel wire, $L$ is the length of steel strands, $u_{0}$ is the vacuum permeability, and $a$ is the twist distance of steel strands.

2.4. Stress-Frequency Relationship. According to material mechanics, the elongation of steel strands under certain stress is as follows:

$$
\begin{aligned}
\Delta l & =\frac{\mathrm{Fl}}{\mathrm{EA}}=\frac{\sigma l}{E}, \\
L & =L_{0}+L_{l}+L_{\sigma} .
\end{aligned}
$$

Because the silver-coated enameled wires are connected at both ends before being connected to the strands, there is a self-contained frequency in the circuit. So, the inductance in the circuit consists of three parts, that is, inherent inductance $L_{0}$, inductance increment $L_{1}$ caused by natural length of steel strands, and stress-induced inductance increment $L_{\sigma} ; L_{0}$ is obtained by connecting the two ends of the conductor in the experiment. The inductance of the inherent length of the strands can be obtained from the following formula:

$$
L_{l}=\frac{u_{0} N^{2} S}{l}=\frac{36 u_{0} l S}{a^{2}}
$$

The inductance increment caused by stress is

$$
L_{\sigma}=-\frac{\Delta l \mu_{0} N^{2} S}{l(l+\Delta l)}=-\frac{36(\sigma / E) \mu_{0} l S}{a^{2}(1+(\sigma / E))} .
$$

Therefore, the resonant frequency can be obtained as follows:

$$
f=\frac{1}{2 \pi \sqrt{\left(L_{0}+\left(36 \mu_{0} l S / a^{2}\right)+\left(-36(\sigma / E) \mu_{0} l S / a^{2}(1+(\sigma / E))\right)\right.} C} .
$$

2.5. Simulation Results. By substituting $\mu_{0}=4 \pi \times 10^{-7} \mathrm{H} / \mathrm{m}$, $\sigma=7.194 \mathrm{MPa}, \quad L_{0}=0.000008 \mathrm{H}, \quad S=139 \times 10^{-6} \mathrm{~m}^{2}$, $a=0.212 \mathrm{~m}, E=1.95 \times 10^{5} \mathrm{MPa}$, and $C=0.1 \mu \mathrm{F}$ into formula (11), the $\sigma-f$ diagram can be obtained, as shown in Figure 2. 


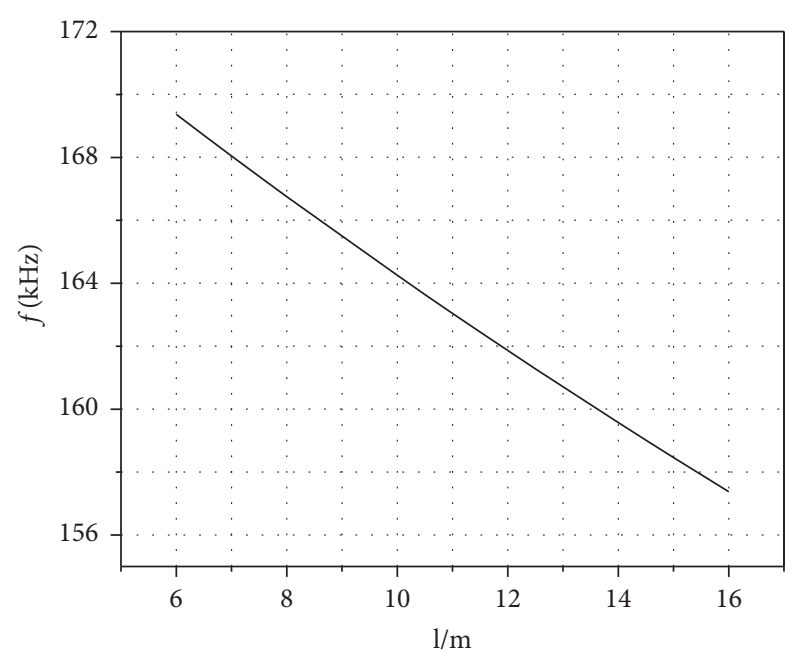

Figure 2: $1-f$ diagram.

$$
f=\frac{1}{2 \pi \sqrt{0.0000008+l \times 1.3984 \times 10^{-8}}} .
$$

By substituting the parameter $l=14 \mathrm{~m}$ into formula (11), the $1-f$ diagram can be obtained as shown in Figure 3.

$$
f=\frac{1}{2 \pi \sqrt{9.958 \times 10^{-7}-\left(1.958 \times 10^{-7} \sigma /(195000+\sigma)\right)}} .
$$

As can be seen from formula (11), the frequency of the LC resonant circuit is related to the stress and natural length of strands. From Figure 3, it can be seen that the resonant frequency of the circuit is positively linearly related to the stress of the strands, and the resonant frequency increases with the increase of stress. As shown in Figure 2, the resonant frequency of the circuit is inversely proportional to the natural length of the strands, and the resonant frequency decreases with the increase of the natural length of the strands. It can be concluded that the stress of the strands in the circuit mainly changes the resonant frequency of the circuit by changing the length of the strands, and the change of relative permeability of steel strands can be ignored. In order to prove this theory, the following experiments are carried out in this paper.

\section{Experiments}

3.1. Experimental Introduction. In order to verify the effect of the change of permeability caused by the stress of strands on the resonant frequency of the LC circuit, experiments were carried out on 7- $\Phi 9 \mathrm{~mm}$ strands $\left(u_{\mathrm{r}}=1500\right)$ and aluminum strands $\left(u_{\mathrm{r}}=1\right)$ with large difference in relative permeability. The circuit frequency of steel strands and aluminum strand tests under the same conditions, respectively, are carried out to determine whether the change of the component permeability will affect the electromagnetic resonance frequency. By testing the circuit frequency of 7$\Phi 15.20 \mathrm{~mm}$ steel strands with different lengths and the same stress, the influence of strand length on electromagnetic

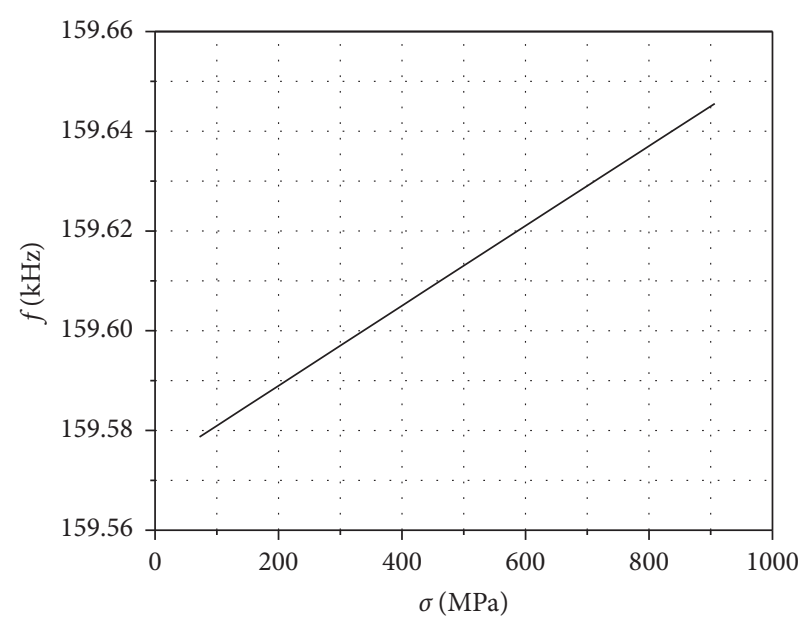

Figure 3: $\sigma-f$ diagram.

resonance frequency of the access circuit is simulated. Finally, the stress-frequency test of $14 \mathrm{~m}$ long $7-\Phi 15.20 \mathrm{~mm}$ steel strands is carried out to verify the revised theory of measuring the stress of steel strands based on the LC resonant circuit.

Schematic diagram of the experimental device for comparative test of steel strands and aluminum strands is shown in Figure 4. The experimental device is divided into two parts: the force transfer device and the test circuit. Loading experiments of specimens with different lengths were carried out by passing the specimens through the reaction frame. Because the reaction frame is fixed on the ground and cannot be moved, it is impossible to obtain enough test data; a force transmission device is made as shown in Figure 4. Two $16 \mathrm{~mm}$ diameter screw rods are used to penetrate the reaction frame. Two $3 \mathrm{~cm}$-thick steel plates are customized and connected with nuts. The screw jack (measuring range is $5 \mathrm{t}$ ) is installed between the outer steel plate and the reaction frame for additional force (Figure 5). The test circuit mainly connects the test piece into the circuit. One end of the test piece fixes the test piece with the steel plate by anchorage, and the other end fixes the test piece on the reaction frame by anchorage (Figure 6). In order to prevent the pressure sensor from falling into the anchor, a cushion plate is installed between the anchor and the pressure sensor. The specimen is connected to the circuit by the silver-plated copper core-enameled wire, and the circuit diagram is shown in Figure 7. Bluetooth is used to transmit the resonant frequency data of the circuit to the computer equipment. The field test drawings are shown in Figure 8.

In order to prevent the reaction frame from introducing the circuit, the PVC tube is used to wrap the test piece at the junction of the test piece and the reaction frame to prevent the contact between the test piece and the reaction frame. The test piece is wound with an insulating tape at the connection of the pressure sensor, steel plate, and cushion plate to prevent the outside from introducing the circuit. In the stress-frequency experiment of $14 \mathrm{~m}$ steel strands, the screw rod cannot bear enough tension, so the transmission device is removed, and the hydraulic jack is used to load the steel strands directly on the reaction frame. The schematic diagram of the experimental device is shown in Figure 9. 


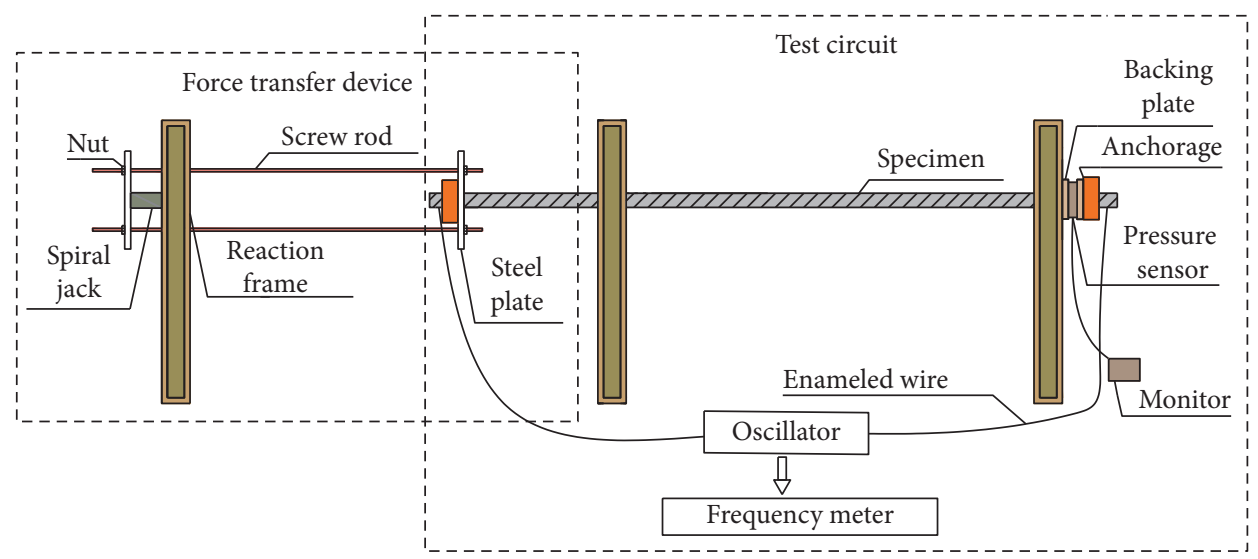

FIgURE 4: Schematic diagram of the experimental device.

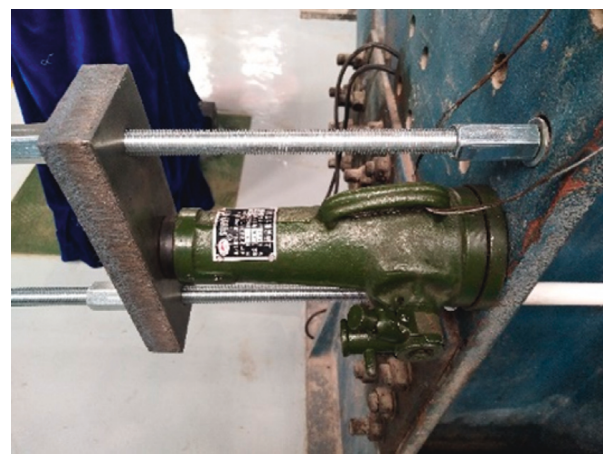

FIGURE 5: Jack apparatus.

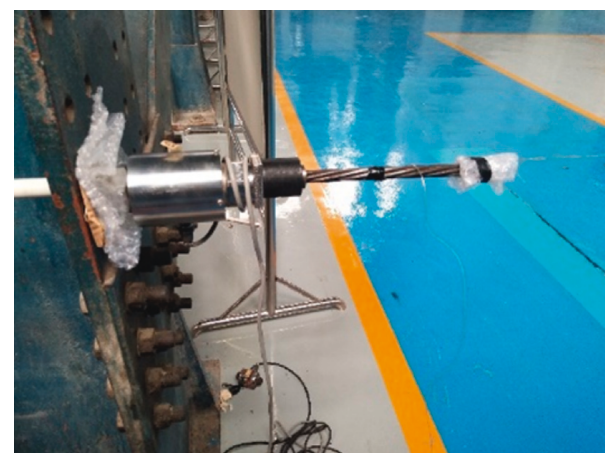

FiguRE 6: Diagram of anchorage, gaskets, and pressure sensor.

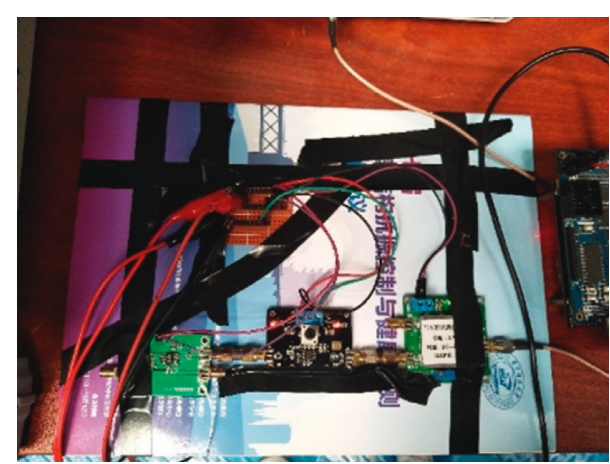

Figure 7: Circuit device diagram.

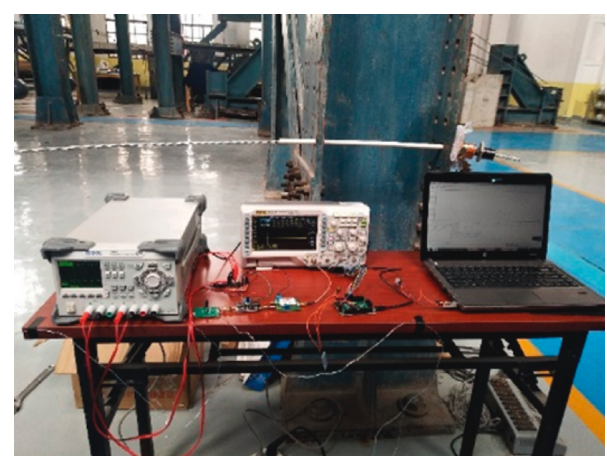

FIGURE 8: Field experimental diagram of aluminum/steel strands.

3.2. Experimental Process. In order to study the effect of permeability on stress detection of strands, the resonant frequency of the same tension force of $9 \mathrm{~mm}$ diameter strands/aluminum strands was measured. Because the maximum tensile stress of the aluminum strands is $7.8 \mathrm{kN}$, considering the deformation and safety factors, the tension force of $1 \mathrm{kN}$ is exerted on the strands. For the sake of the authenticity of the experimental data, many groups of experiments were carried out, so experiments were carried out on steel strands/aluminum strands with different lengths, ranging from $600 \mathrm{~cm}$ to $1650 \mathrm{~cm}$.

Before the experiment, the experimental equipment is debugged, and the pressure sensor is calibrated. The 


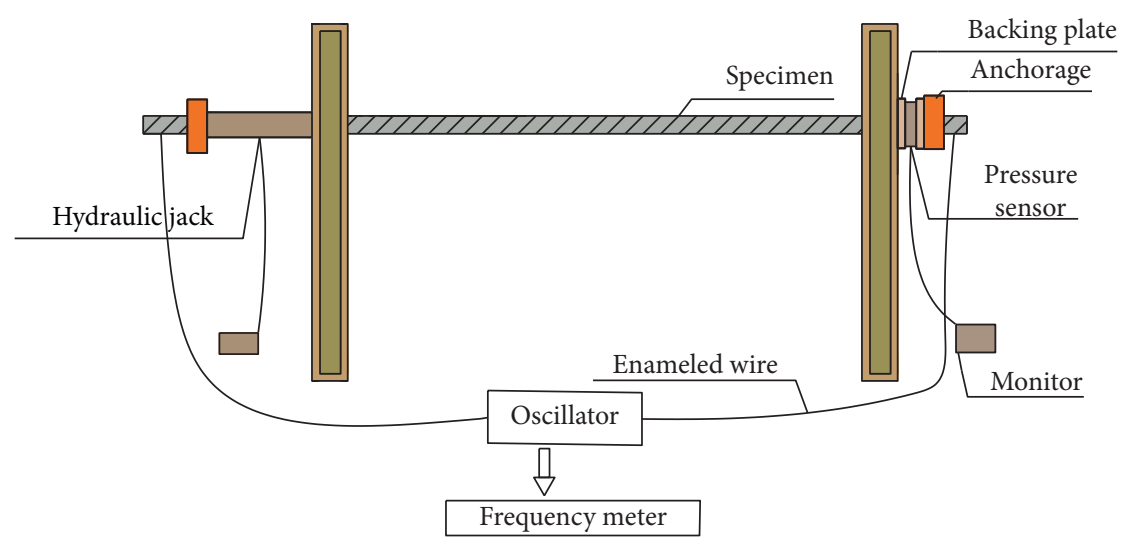

Figure 9: $6 \mathrm{~m} / 10 \mathrm{~m} / 14 \mathrm{~m}$ experimental device of steel strands.

TABLE 1: Table of experimental parameters.

\begin{tabular}{lccc}
\hline Parameter & Permeability & Length $(\mathrm{m})$ & Stress $(\mathrm{MPa})$ \\
\hline Numerical value & $7-\Phi 9 \mathrm{~mm}$ steel/aluminum strands & $16.5,16, \ldots, 6$ & $100,200, \ldots, 900$ \\
Note appended & $u_{\mathrm{r}} \approx 1500, u_{\mathrm{r}} \approx 1$, see Figure 10 & See Figure 11 & See Figure 12 \\
\hline
\end{tabular}

sensitivity coefficient of the pressure sensor connecting the strain box is set to 0.3846 . When the specimen is relaxed, the display of the strain box is set to 0 . When the tension is $1 \mathrm{kN}$, the resonant frequency of the circuit is recorded, and each group of experiments is repeated three times. The same experiment was carried out on steel strands with diameter of $15.20 \mathrm{~mm}$.

In order to analyze the stress-frequency relationship, the stress-frequency experiment of $14 \mathrm{~m}$-long $7-\Phi 15.20 \mathrm{~mm}$ strands was carried out, and the tension was controlled from $10 \mathrm{kN}$ to $126 \mathrm{kN}$. The resonant frequencies of the circuits under different stresses were measured, and the experiments were repeated four times in each group. Detailed changes in parameters are shown in Table 1.

3.3. Experimental Data. In order to study whether the change of stress-relative permeability will affect the resonant frequency of the circuit, experiments were carried out on 7 $\Phi 9 \mathrm{~mm}$ steel strands and aluminum strands with length of $6 \mathrm{~m}-16 \mathrm{~m}$. The experimental data are shown in Tables 2 and 3 , and the analysis results are shown in Figure 10.

In order to study the influence of strand length on circuit resonance frequency, experiments of different lengths of 7$\Phi 15.20 \mathrm{~mm}$ strands commonly used in engineering are carried out, ranging from $6-16 \mathrm{~m}$. The experimental data are shown in Table 4, and the analysis drawings are shown in Figure 11.

In order to study the effect of stress on resonance frequency and verify the theoretical formula, the $\sigma-f$ experiment of $14 \mathrm{~m} 7-\Phi 15.20 \mathrm{~mm}$ steel strands is carried out. The experimental data are shown in Table 5, and the analysis diagram is shown in Figure 12.

\subsection{Data Analysis}

(1) When the tension force of $\Phi 9 \mathrm{~mm}$ steel strands is measured at $1 \mathrm{kN}$, the error rate of data repetition is controlled at $0.038 \%$. Under the same conditions, the data repetition error rate of $\Phi 9 \mathrm{~mm}$ diameter aluminum strands is controlled at $0.047 \%$ and that of $\Phi 15.20 \mathrm{~mm}$ diameter steel strands is controlled at $0.045 \%$. The steel strands with $14 \mathrm{~m}$ length are tested in $\sigma$ - $f$ experiment, and data duplication error rate is only $0.010 \%$.

(2) As shown in Figure 10, $\Phi 9 \mathrm{~mm}$ steel strands and aluminum strands access the circuit, and the resonant frequency of steel strands/aluminum strands with the same stress and length tends to be the same. When the length is $6 \mathrm{~m}$, the maximum frequency difference is $1 \mathrm{kHz}$, and the frequency difference of other length specimens is very small. Error data when $6 \mathrm{~m}$ are considered. At the same time, the frequency of strands/aluminum strands is consistent with the length, that is, it decreases with the increase of length. Because the permeability of steel strands $\left(u_{\mathrm{r}}=1500\right)$ and aluminum strands $\left(u_{\mathrm{r}}=1\right)$ is quite different, therefore, both theoretical and experimental data are available. If other conditions are consistent, the difference of relative permeability of strands itself has little effect on resonance frequency. It can be concluded that the effect of stress changerelative permeability on resonance frequency is smaller.

(3) Figure 11 shows that under the same stress, the resonance frequency of $\Phi 15.20 \mathrm{~mm}$ strands changes with the length; the frequency decreases linearly with the increase of length, and it is consistent with that of $\Phi 9 \mathrm{~mm}$ steel strands/aluminum strands varying with length. Comparing $1-f$ analysis with Figures 2 and 11, we can see that, under the same stress, the resonance frequency of different length strands decreases with the increase of length, and the experimental results are consistent with the theoretical ones. 
TABLe 2: $\Phi 9 \mathrm{~mm}$ experimental data of aluminum strands.

\begin{tabular}{lccccccc}
\hline$l / \mathrm{m}$ & $f 1(\mathrm{kHz})$ & $f 2(\mathrm{kHz})$ & $f 3(\mathrm{kHz})$ & $f 4(\mathrm{kHz})$ & $\mathrm{SD}$ & $\mathrm{RE}(\%)$ \\
\hline 6.0 & 104.612 & 104.625 & 104.606 & 104.608 & 0.0086 & 0.0182 \\
6.5 & 103.273 & 103.277 & 103.262 & 103.281 & 0.0082 & 0.0184 \\
7.0 & 101.836 & 101.847 & 101.864 & 101.856 & 0.0122 & 0.0278 \\
7.5 & 101.166 & 101.179 & 101.191 & 101.196 & 0.0134 & 0.0296 \\
8.0 & 100.815 & 100.784 & 100.787 & 100.794 & 0.0140 & 0.0308 \\
10.0 & 98.421 & 98.363 & 98.465 & 98.397 & 0.0426 & 0.1030 \\
10.5 & 97.621 & 97.666 & 97.643 & 97.633 & 0.0192 & 0.0464 \\
11.0 & 97.009 & 97.014 & 97.018 & 96.994 & 0.0106 & 0.0247 \\
11.5 & 96.350 & 96.326 & 96.339 & 96.365 & 0.0166 & 0.0405 \\
12.0 & 95.704 & 95.707 & 95.719 & 95.705 & 0.0072 & 0.0164 \\
12.5 & 94.965 & 94.938 & 94.934 & 94.951 & 0.0140 & 0.0326 \\
14.0 & 94.186 & 94.199 & 94.171 & 94.184 & 0.0115 & 97.031 \\
14.5 & 93.541 & 93.566 & 93.542 & 93.547 & 0.0117 & 96.0297 \\
15.0 & 93.219 & 93.228 & 93.210 & 93.222 & 0.0076 & 0.0267 \\
15.5 & 92.676 & 92.680 & 92.665 & 92.676 & 0.0064 & 0.0197 \\
16.0 & 92.480 & 92.448 & 92.492 & 92.465 & 0.0188 & 0.0158 \\
\hline
\end{tabular}

TABle 3: $\Phi 9 \mathrm{~mm}$ experimental data of steel strands.

\begin{tabular}{|c|c|c|c|c|c|c|c|}
\hline$l / \mathrm{m}$ & $f 1(\mathrm{kHz})$ & $f 2(\mathrm{kHz})$ & $f 3(\mathrm{kHz})$ & $f 4(\mathrm{kHz})$ & SD & RE (\%) & $f(\mathrm{kHz})$ \\
\hline 6.0 & 103.813 & 103.819 & 103.815 & 103.805 & 0.0057 & 0.0128 & 103.813 \\
\hline 6.5 & 101.991 & 102.009 & 102.035 & 102.013 & 0.0180 & 0.0428 & 102.012 \\
\hline 7.0 & 102.379 & 102.378 & 102.385 & 102.374 & 0.0047 & 0.0111 & 102.379 \\
\hline 7.5 & 101.660 & 101.663 & 101.662 & 101.653 & 0.0044 & 0.0098 & 101.660 \\
\hline 8.0 & 100.993 & 101.011 & 101.023 & 101.026 & 0.0149 & 0.0327 & 101.013 \\
\hline 10.0 & 99.016 & 99.044 & 99.043 & 99.042 & 0.0136 & 0.0283 & 99.036 \\
\hline 10.5 & 98.125 & 98.121 & 98.126 & 98.132 & 0.0047 & 0.0115 & 98.126 \\
\hline 11.0 & 97.460 & 97.471 & 97.473 & 97.474 & 0.0068 & 0.0150 & 97.470 \\
\hline 11.5 & 95.727 & 95.715 & 95.726 & 95.739 & 0.0098 & 0.0251 & 95.727 \\
\hline 12.0 & 94.963 & 94.976 & 94.973 & 94.981 & 0.0073 & 0.0183 & 94.973 \\
\hline 12.5 & 94.376 & 94.371 & 94.378 & 94.379 & 0.0035 & 0.0081 & 94.376 \\
\hline 14.0 & 93.786 & 93.790 & 93.789 & 93.792 & 0.0023 & 0.0060 & 93.789 \\
\hline 14.5 & 93.520 & 93.528 & 93.539 & 93.534 & 0.0081 & 0.0203 & 93.530 \\
\hline 15.0 & 92.771 & 92.786 & 92.774 & 92.794 & 0.0106 & 0.0244 & 92.781 \\
\hline 15.5 & 92.658 & 92.655 & 92.653 & 92.674 & 0.0096 & 0.0227 & 92.660 \\
\hline 16.0 & 92.415 & 92.422 & 92.450 & 92.434 & 0.0152 & 0.0375 & 92.430 \\
\hline
\end{tabular}

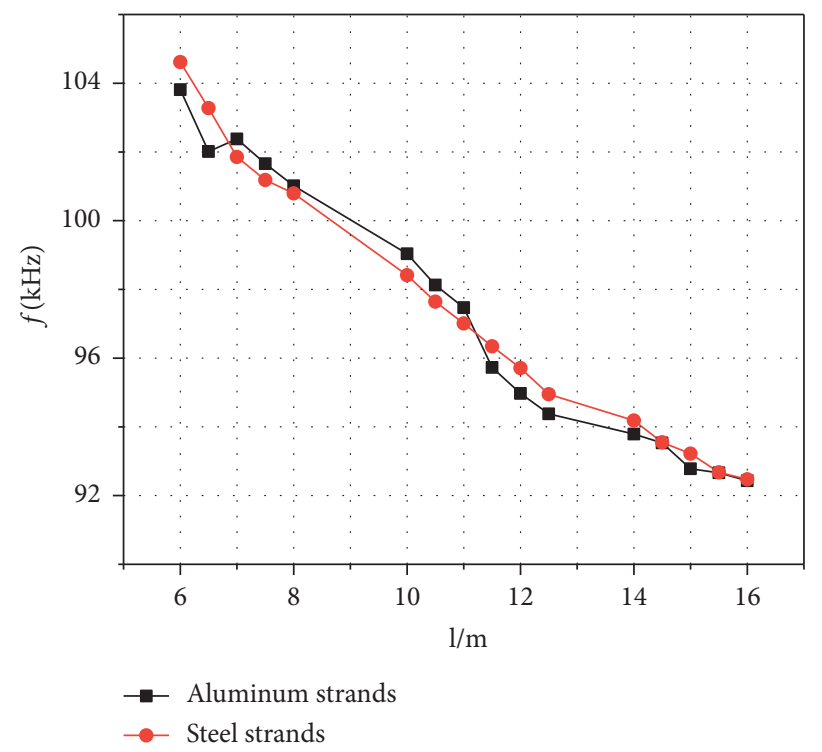

FIGURE 10: $1-f$ analysis diagram of steel strands/aluminum strands. 
TABLe 4: $\Phi 15.2 \mathrm{~mm}$ experimental data of strand length.

\begin{tabular}{|c|c|c|c|c|c|c|c|}
\hline$l / \mathrm{m}$ & $f 1(\mathrm{kHz})$ & $f 2(\mathrm{kHz})$ & $f 3(\mathrm{kHz})$ & $f 4(\mathrm{kHz})$ & SD & $\mathrm{RE}(\%)$ & $f(\mathrm{kHz})$ \\
\hline 6.0 & 103.907 & 103.909 & 103.900 & 103.912 & 0.0051 & 0.0115 & 103.907 \\
\hline 6.5 & 103.183 & 103.183 & 103.213 & 103.225 & 0.0214 & 0.0407 & 103.201 \\
\hline 7.0 & 102.522 & 102.536 & 102.538 & 102.540 & 0.0082 & 0.0176 & 102.534 \\
\hline 7.5 & 101.394 & 101.395 & 101.372 & 101.379 & 0.0113 & 0.0227 & 101.385 \\
\hline 8.0 & 100.745 & 100.734 & 100.753 & 100.748 & 0.0080 & 0.0189 & 100.745 \\
\hline 10.0 & 98.675 & 98.641 & 98.652 & 98.648 & 0.0147 & 0.0345 & 98.654 \\
\hline 10.5 & 97.485 & 97.462 & 97.502 & 97.479 & 0.0165 & 0.0410 & 97.482 \\
\hline 11.0 & 97.287 & 97.293 & 97.285 & 97.283 & 0.0043 & 0.0103 & 97.287 \\
\hline 11.5 & 96.640 & 96.643 & 96.642 & 96.635 & 0.0036 & 0.0083 & 96.640 \\
\hline 12.0 & 95.778 & 95.784 & 95.776 & 95.774 & 0.0043 & 0.0104 & 95.778 \\
\hline 12.5 & 95.482 & 95.446 & 95.447 & 95.485 & 0.0214 & 0.0409 & 95.465 \\
\hline 14.0 & 94.153 & 94.139 & 94.162 & 94.158 & 0.0100 & 0.0244 & 94.153 \\
\hline 14.5 & 93.705 & 93.698 & 93.695 & 93.714 & 0.0084 & 0.0203 & 93.703 \\
\hline 15.0 & 93.138 & 93.164 & 93.127 & 93.123 & 0.0185 & 0.0440 & 93.138 \\
\hline 15.5 & 92.490 & 92.476 & 92.512 & 92.470 & 0.0187 & 0.0454 & 92.487 \\
\hline 16.0 & 92.211 & 92.202 & 92.200 & 92.223 & 0.0105 & 0.0249 & 92.209 \\
\hline
\end{tabular}

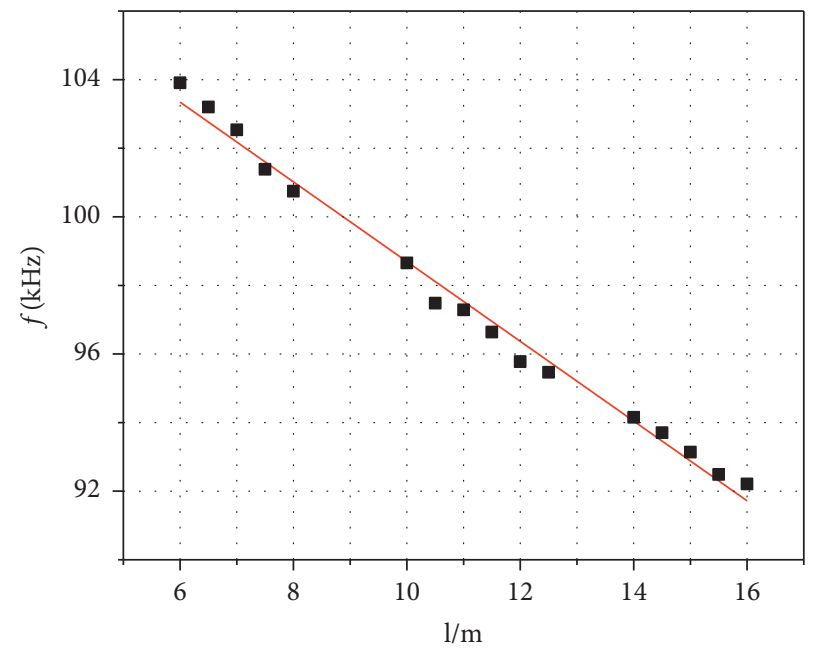

FIGURE 11: 1- $f$ analysis diagram of steel strands.

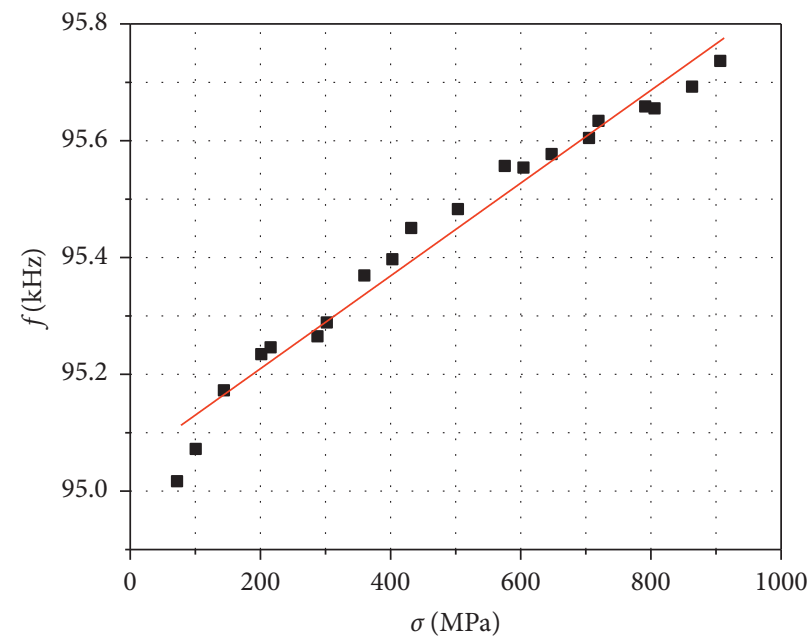

FIGURE 12: $14 \mathrm{~m}$ steel strand $\sigma-f$ analysis chart. 
TABLE 5: $14 \mathrm{~m} \Phi 15.20 \mathrm{~mm}$ experimental data of steel strands.

\begin{tabular}{|c|c|c|c|c|c|c|c|}
\hline$\sigma(\mathrm{Mpa})$ & $f 1(\mathrm{kHz})$ & $f 2(\mathrm{kHz})$ & f3 $(\mathrm{kHz})$ & $f 4(\mathrm{kHz})$ & SD & RE (\%) & $f(\mathrm{kHz})$ \\
\hline 71.94 & 95.007 & 95.024 & 95.012 & 95.025 & 0.0089 & 0.0189 & 95.017 \\
\hline 100.72 & 95.073 & 95.066 & 95.080 & 95.070 & 0.0059 & 0.0147 & 95.072 \\
\hline 143.88 & 95.169 & 95.166 & 95.177 & 95.178 & 0.0059 & 0.0126 & 95.173 \\
\hline 201.44 & 95.232 & 95.236 & 95.231 & 95.239 & 0.0037 & 0.0084 & 95.235 \\
\hline 215.83 & 95.250 & 95.241 & 95.248 & 95.246 & 0.0039 & 0.0094 & 95.246 \\
\hline 287.77 & 95.270 & 95.266 & 95.260 & 95.264 & 0.0042 & 0.0105 & 95.265 \\
\hline 302.16 & 95.279 & 95.290 & 95.293 & 95.293 & 0.0067 & 0.0147 & 95.289 \\
\hline 359.71 & 95.369 & 95.371 & 95.361 & 95.376 & 0.0062 & 0.0157 & 95.369 \\
\hline 402.88 & 95.396 & 95.392 & 95.397 & 95.403 & 0.0045 & 0.0115 & 95.397 \\
\hline 431.65 & 95.446 & 95.451 & 95.453 & 95.452 & 0.0031 & 0.0073 & 95.451 \\
\hline 503.60 & 95.484 & 95.480 & 95.481 & 95.486 & 0.0028 & 0.0063 & 95.483 \\
\hline 575.54 & 95.568 & 95.564 & 95.568 & 95.567 & 0.0019 & 0.0042 & 95.567 \\
\hline 604.32 & 95.554 & 95.551 & 95.553 & 95.558 & 0.0029 & 0.0073 & 95.554 \\
\hline 647.48 & 95.575 & 95.574 & 95.579 & 95.580 & 0.0029 & 0.0063 & 95.577 \\
\hline 705.04 & 95.606 & 95.599 & 95.605 & 95.608 & 0.0039 & 0.0094 & 95.605 \\
\hline 719.42 & 95.637 & 95.630 & 95.634 & 95.635 & 0.0029 & 0.0073 & 95.634 \\
\hline 791.37 & 95.657 & 95.656 & 95.658 & 95.663 & 0.0031 & 0.0073 & 95.659 \\
\hline 805.76 & 95.659 & 95.655 & 95.647 & 95.660 & 0.0059 & 0.0136 & 95.655 \\
\hline 863.31 & 95.693 & 95.692 & 95.698 & 95.687 & 0.0045 & 0.0115 & 95.693 \\
\hline 906.47 & 95.726 & 95.733 & 95.756 & 95.731 & 0.0133 & 0.0313 & 95.737 \\
\hline
\end{tabular}

(4) Figure 12 shows that the resonance frequency of $14 \mathrm{~m} \Phi 15.20 \mathrm{~mm}$ steel strands increases with the increase of stress. There is a good positive linear correlation between stress and frequency. The data fitting equation is as follows: $f=7.94606 \times 10^{-4} \sigma+95.0528, \quad R^{2}=0.96752$. Comparing with Figure 3, it can be found that there is a consistent correlation between experimental data and theoretical data in stress and frequency. But, the slope of fitting formula is 10 times that of theoretical formula. Considering that, under the action of stress, while the length of strands changes, the compactness of seven steel wires in the strand structure changes. Therefore, the increase of the length produced by the stress cannot be simply equal to the increase of the length of the strands. Further research is needed in the next study.

(5) The theoretical and experimental data show that compared with the influence of specimen length on relative permeability, the influence of relative permeability on resonance frequency can be ignored. The smaller the natural length of strands is, the more significant the resonance frequency is. Therefore, it can be deduced that the effect of stress-changing permeability on resonance frequency can be ignored. The stress mainly changes resonance frequency by changing the length of steel strands.

\section{Conclusions}

The stress loss of the prestressed steel strands of the bridges in service makes the deflection of the structure to decrease and the main beam to crack, which seriously threaten the bearing capacity of the structure. It is of great significance to realize the detection of steel strand prestress. LC resonance connects the steel strands as an inductance through the conductor into the circuit and realizes the stress detection of the prestressed steel strands by testing the resonance frequency of the circuit. The previous research team has proved that the resonance frequency of the circuit has a good linear correlation with the stress of the steel strands, and this method has great potential for the stress detection of the prestressed steel strands. As a kind of magnetic material, the magnetic properties of the steel strands will change when it deforms under the stress. Therefore, it is very important to know which parameters affect the resonant frequency of the stress through changing. In this paper, the influence of permeability and length parameters on the resonant frequency of the circuit is studied in order to provide guidance for the future prestressing detection method.

In this paper, the relationship between stress and relative permeability and length is analyzed theoretically. The theory of stress detection of prestressed steel strands based on the LC resonant circuit is modified and verified by experiments. The inductance in the circuit is simplified into three parts: the inherent inductance generated by the circuit, the inductance caused by different lengths of steel strands, and the inductance increment caused by stress change.

Through theoretical analysis, it is found that the length is the main influence factor of resonance frequency. The theory is verified by establishing experiments of different lengths of steel strands and aluminum strands; according to the experiment, compared with the effect of length, the change of resonance frequency caused by the change of stress on permeability can be ignored. The stress mainly changes the resonance through the change of length; the resonance frequency has a negative linear correlation with the natural length, and the frequency decreases linearly with the increase of the natural length, and the experimental data are consistent with the theoretical law; the frequency of a certain length of steel strands is proportional to the stress, and the 
stress is linearly related to the frequency. The law of experimental data is consistent with the theoretical derivation.

In the next research, we need to further study the influence of the length change of the stress on the resonant frequency. In addition, the research on the stress frequency of the prestressed concrete structure with bond is carried out to make further efforts to realize the stress detection of the steel strands of the prestressed concrete bridges in service.

\section{Data Availability}

The data used to support the findings of this study are included within the article.

\section{Conflicts of Interest}

The authors declare that there are no conflicts of interest regarding the publication of this paper.

\section{Acknowledgments}

This research was funded by the National Key Research and Development Program of China (grant no. 2017YFC0806007), the Program for Innovation Team Building at Institutions of Higher Education in Chongqing (grant no. CXTDG201602013), the Technology Innovation Project of Chongqing Social Undertakings and People's Livelihood (grant no. CSTC2016SHMSZX30026), the Urumqi Science and Technology Plan (grant no. Y161320008), and the Key Technology Research Project for construction of the extrahigh pier T-shaped bridge in high strength and high altitude area from Xinjiang Communications Construction Group Co., Ltd.

\section{References}

[1] K. J. Shin, S. Cheol Lee, Y. Kim, J.-M. Kim, S. Park, and H. Lee, "Construction condition and damage monitoring of posttensioned psc girders using embedded sensors," Sensors, vol. 17, no. 8, 2017.

[2] T. C. Huynh and J. T. Kim, "Fos-based prestress force monitoring and temperature effect estimation in unbonded tendons of psc girders," Journal of Aerospace Engineering, vol. 30, no. 2, pp. 1-14, 2016.

[3] C. Travaglini, C. Bescond, M. Viens, and P. Belanger, "Feasibility of high frequency guided wave crack monitoring," Structural Health Monitoring, vol. 16, no. 4, pp. 418-427, 2017.

[4] R. Singh, S. Sharm, and S. Sharma, "Guided waves for damage monitoring in plates with corrosion defects," International Journal of Innovative Research in Science, Engineering and Technology, vol. 3, no. 3, 2014.

[5] J. Qian, X. Chen, L. Sun, G. Yao, and XuWang, "Numerical and experimental identification of seven-wire strands tensions using scale energy entropy spectra of ultrasonic guided waves," Shock and Vibration, vol. 2018, Article ID 6905073, 11 pages, 2018.

[6] W. Yin, X. Zhou, Y. Duan, K. Fan, Ru Zhang, and Z. Yang, "Elasto-magnetic effect based stress monitoring of highstrength steel wires:experimental investigation," Structural Engineers, vol. 29, pp. 113-118, 2013.

[7] G. Pang, C. Dong, Y. Duan, and Z. chaobo, "Elasto-magnetic effect based total stress monitoring of prestressed steel strands: experimental investigation," Structural Engineers, vol. 31, pp. 192-198, 2015.

[8] Ru Zhang, Y. Duan, W. Siu, and Y. Zhao, "Smart elastomagneto-electric (EME) sensors for stress monitoring of steel cables: design theory and experimental validation," Sensors, vol. 14, no. 8, pp. 13644-13660, 2014.

[9] C. Dong, L. Guo, G. Pang, H. Chen, Y. Duan, and C. Zhou, "Elasto-magnetic effect based total stress monitoring of internal prestressing tendons:experimental investigation and engineering application," Structural Engineers, vol. 32, pp. 74-79, 2016.

[10] H. Zhang, Z. Tian, Y. Duan, C. Zhou, and F. Li, "Elastomagnetic effect based suspender force monitoring of an arch bridge," Structural Engineers, vol. 32, pp. 80-84, 2016.

[11] Z. Tian, H. Zhang, Y. Luo, Y. Duan, C. Zhou, and Q. Chen, "Elasto-magnetic effect based total stress monitoring of screwthread steel," Structural Engineers, vol. 33, pp. 111-116, 2017.

[12] C. Joh, J. W. Lee, and I. Kwahk, "Feasibility study of stress measurement in prestressing tendons using villari effect and induced magnetic field," International Journal of Distributed Sensor Networks, vol. 9, no. 11, 2013.

[13] J. Kim, J. W. Kim, C. Lee, and S. Park, "Development of embedded em sensors for estimating tensile forces of psc girder bridges," Sensors, vol. 17, no. 9, 2017.

[14] D. Zhou, M. Pan, Y. He, and B. Du, "Stress detection and measurement in ferromagnetic metals using pulse electromagnetic method with U-shaped sensor," Measurement, vol. 105, pp. 136-145, 2017.

[15] D. Chen, B. Zhang, X. Li et al., "A stress measurement method for steel strands based on LC oscillation," Advances in Materials Science and Engineering, vol. 2018, Article ID 1584903, 8 pages, 2018.

[16] X. Li, B. Zhang, C. Yuan, T. Chong, D. Chen et al., "An electromagnetic oscillation method for stress measurement of steel strands," Measurement, vol. 125, pp. 330-335, 2018.

[17] B. Zhang, C. Tu, X. Li, H. Cui, and G. Zheng, "Length effect on the stress detection of prestressed steel strands based on electromagnetic oscillation method," Sensors, vol. 19, no. 12, 2019. 


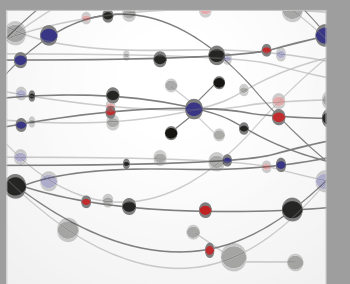

The Scientific World Journal
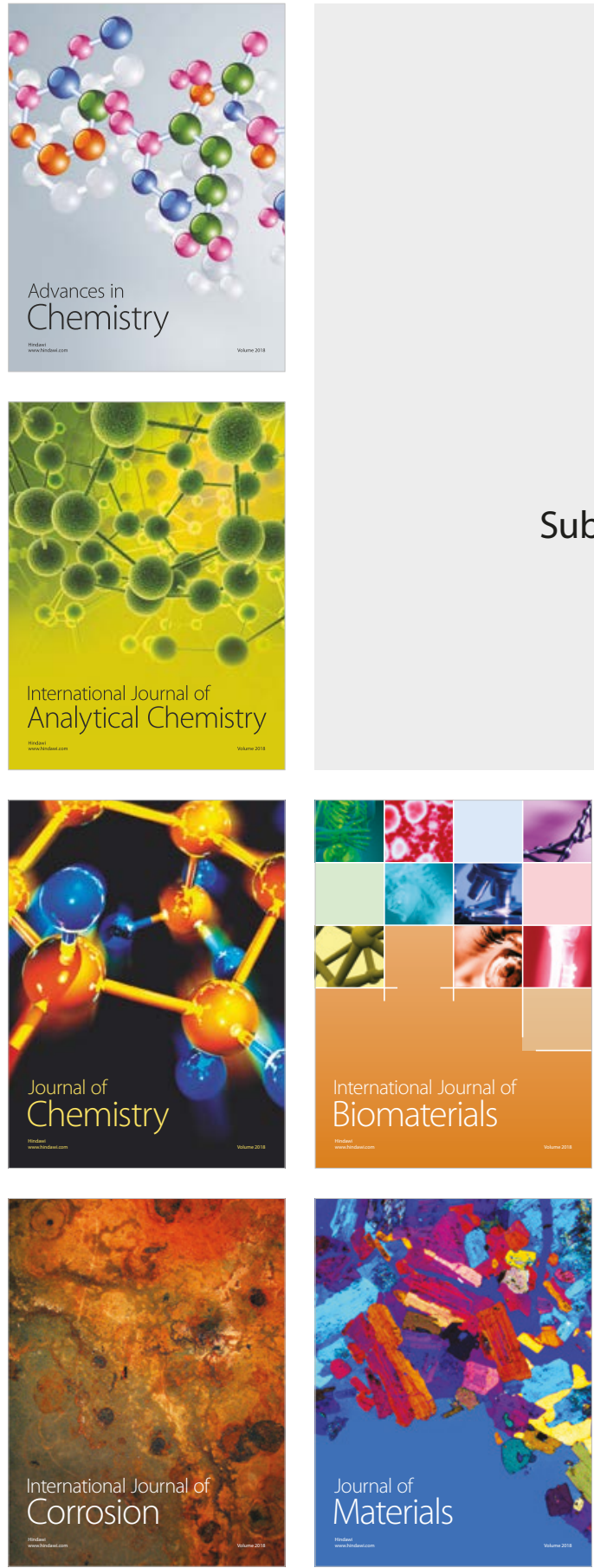

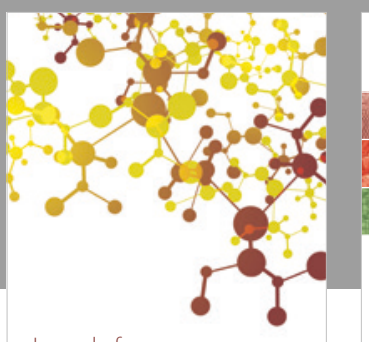

Journal of

Applied Chemistry
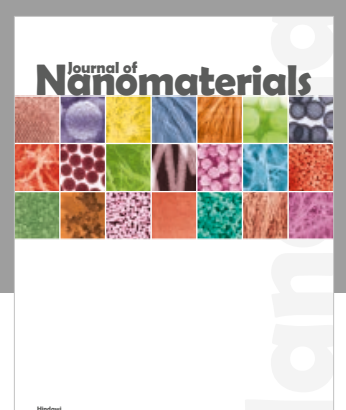

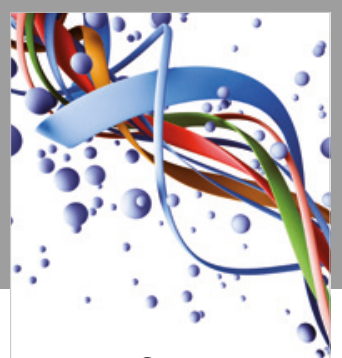

Scientifica

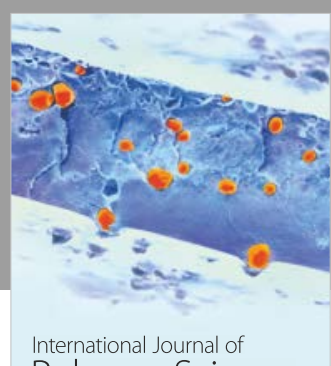

Polymer Science

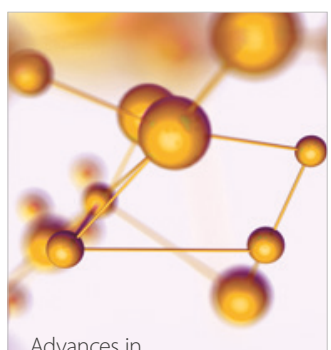

Physical Chemistry
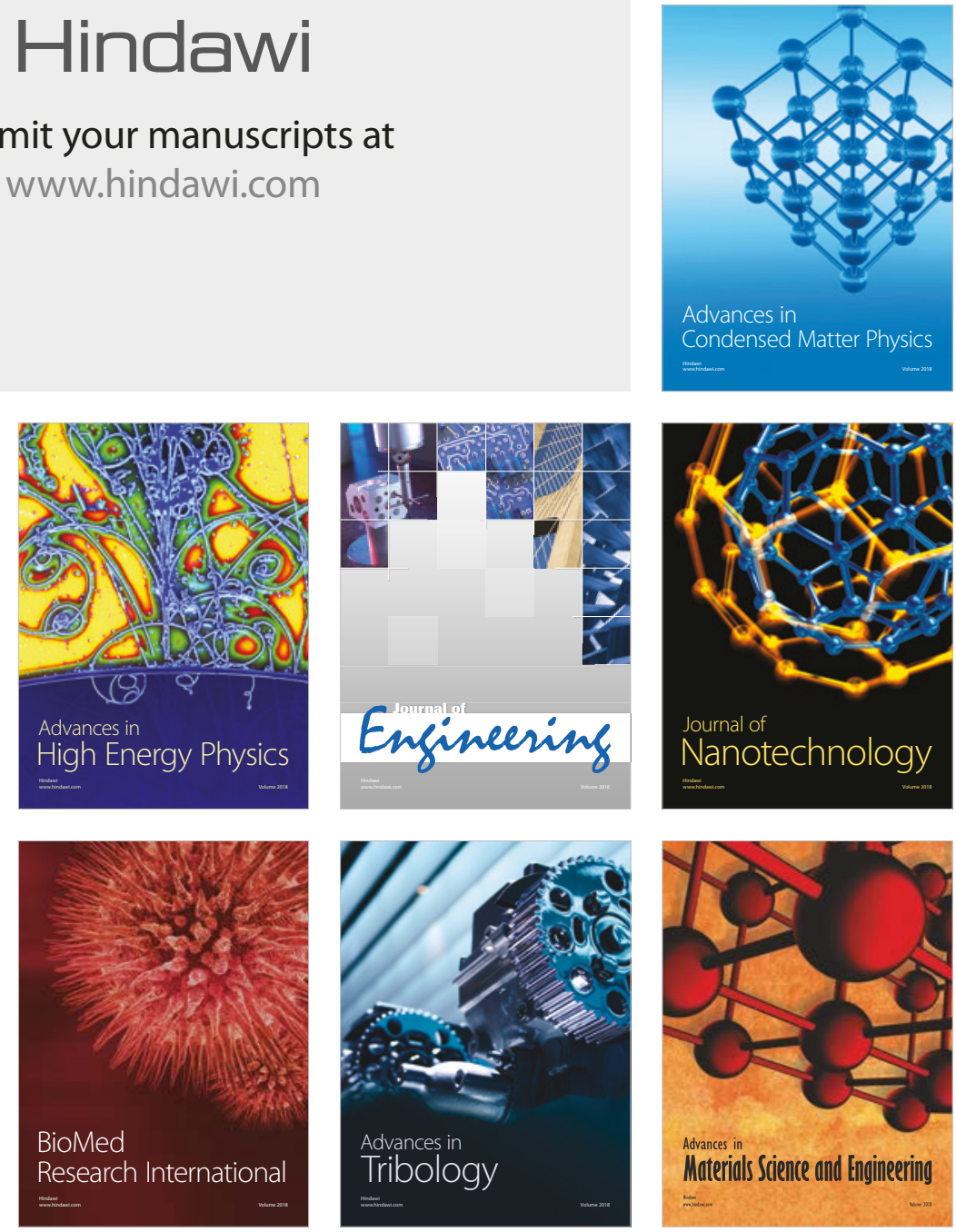